\title{
Irreversible Work and Inner Friction in Quantum Thermodynamic Processes
}

\author{
F. Plastina, ${ }^{1,2}$ A. Alecce, ${ }^{3}$ T. J. G. Apollaro, ${ }^{1,2,4}$ G. Falcone, ${ }^{1,2}$ G. Francica, ${ }^{1,2}$ F. Galve, ${ }^{5}$ N. Lo Gullo, ${ }^{3,6}$ and R. Zambrini ${ }^{5}$ \\ ${ }^{1}$ Dip. Fisica, Università della Calabria, 87036 Arcavacata di Rende (CS), Italy \\ ${ }^{2}$ INFN-Gruppo collegato di Cosenza, Cosenza, Italy \\ ${ }^{3}$ Dipartimento di Fisica e Astronomia "G. Galilei”, Università degli Studi di Padova, via Marzolo 8, 35131 Padova (Italy) \\ ${ }^{4}$ Centre for Theoretical Atomic, Molecular and Optical Physics, School of Mathematics and Physics, \\ Queen's University, Belfast BT7 INN, United Kingdom \\ ${ }^{5}$ IFISC (UIB-CSIC), Instituto de Física Interdisciplinar y Sistemas Complejos, UIB Campus, E-07122 Palma de Mallorca, Spain \\ ${ }^{6}$ CNISM, Sezione di Padova, Italy
}

(Received 4 August 2014; published 31 December 2014)

\begin{abstract}
We discuss the thermodynamics of closed quantum systems driven out of equilibrium by a change in a control parameter and undergoing a unitary process. We compare the work actually done on the system with the one that would be performed along ideal adiabatic and isothermal transformations. The comparison with the latter leads to the introduction of irreversible work, while that with the former leads to the introduction of inner friction. We show that these two quantities can be treated on an equal footing, as both can be linked with the heat exchanged in thermalization processes and both can be expressed as relative entropies. Furthermore, we show that a specific fluctuation relation for the entropy production associated with the inner friction exists, which allows the inner friction to be written in terms of its cumulants.
\end{abstract}

With the increasing ability to manufacture and control microscopic systems, we are approaching the limit where quantum fluctuations, as well as thermal ones, become important when trying to put nanomachines and quantum engines to useful purposes [1,2]. To discuss engine performance, e.g., for heat-to-work conversion, one typically starts by considering reversible transformations that drive the system from an equilibrium configuration to another one. However, if the system is pushed faster than the thermalization time, such transformations are irreversible, and can lead outside the manifold of equilibrium states [3-5]. Nonetheless, these processes are of interest as the reversible protocols, despite enjoying very good efficiencies, give rise to very small output power [6]. The irreversibility of a process is hence related to both better performance and lack of control, leading to entropy production [7].

To analyze irreversibility and entropy production in the quantum realm, we consider a system initially kept in equilibrium and subject to a finite time adiabatic transformation. While its initial state is prepared by keeping it in contact with a thermal bath, the system is then thermally isolated and subject to a parametric change of its Hamiltonian from an initial $H_{i}=H\left[\lambda_{i}\right]$ to a final $H_{f}=$ $H\left[\lambda_{f}\right]$ in a finite time $\tau$. The process is defined by the time variation of the work parameter $\lambda(t)$, changing from $\lambda(t=0)=\lambda_{i}$ to $\lambda(\tau)=\lambda_{f}$.

The work $w$ performed on the system during such a process is a stochastic variable with an associated probability density $p(w)[4,8,9]$, which can be reconstructed experimentally $[10,11]$ through the characteristic function [12]. The fluctuations of work are constrained by the Jarzynski relation [8]

$$
\left\langle e^{-\beta_{i} w}\right\rangle=e^{-\beta_{i} \Delta F},
$$

where $\beta_{i} \equiv \beta_{B}$ is the initial inverse temperature, while $\Delta F=$ $F\left[\lambda_{f}, \beta_{B}\right]-F\left[\lambda_{i}, \beta_{i}\right]$ is the free energy difference between two equilibrium configurations (at the same temperature) corresponding to the initial and final Hamiltonian. This fluctuation relation encodes the full nonlinear response of a system to a time-dependent change of its Hamiltonian. Through the use of the Jensen's inequality, this relation implies that $\langle w\rangle \geq \Delta F$. This, in turn, leads to the introduction of the so-called average irreversible work [13], $\left\langle w_{\text {irr }}\right\rangle=\langle w\rangle-\Delta F \geq 0$. Loosely speaking, $\left\langle w_{\text {irr }}\right\rangle$ gives a measure of the irreversibility introduced by performing the unitary transformation $U(\tau, 0)$, generated by the Hamiltonian $H[\lambda(t)]$ between $t=0$ and $t=\tau$.

The situation is sketched in Fig. (1), where the point $i$ corresponds to the initial state $\rho_{i}=e^{-\beta_{i} H_{i}} / Z\left[\lambda_{i}, \beta_{i}\right]$, while the point $\tau$ corresponds to the state $\rho_{\tau}=U(\tau, 0) \rho_{i} U^{\dagger}(\tau, 0)$. Notice that this latter point does not lay on the manifold of equilibrium states (it could do so only in the limit $\tau \rightarrow \infty$, in which the transformation would become quasistatic).

It has been shown in Ref. [14] that $\left\langle w_{\text {irr }}\right\rangle$ is given by the distance between the actual final state $\rho_{\tau}$ and the (hypothetical) equilibrium state $\rho_{B}=e^{-\beta_{B} H_{f}} / Z(B)$, evaluated through the quantum relative entropy

$$
\left\langle w_{\text {irr }}\right\rangle=\frac{1}{\beta_{B}} D\left(\rho_{\tau} \| \rho_{B}\right) .
$$

The irreversible work has been used to learn about the amount of irreversibility of a given process in a variety of cases, ranging from simple harmonic systems [15], to spin chains [16,17], and ultracold gases [18]. 


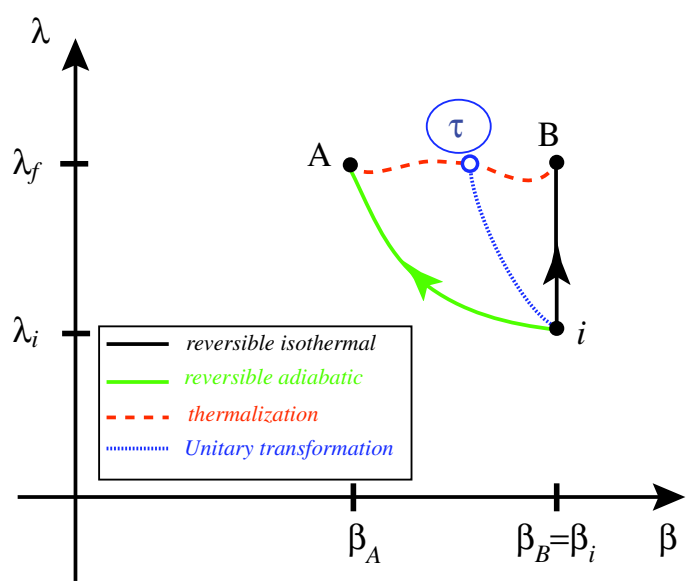

FIG. 1 (color online). Sketch of the transformations considered. The full black circles represent equilibrium states $\rho_{i}=Z_{i}^{-1} e^{-\beta_{i} H_{i}}$ $\rho_{A}=Z_{A}^{-1} e^{-\beta_{A} H_{f}}$, and $\rho_{B}=Z_{B}^{-1} e^{-\beta_{B} H_{f}}$. The empty blue one, instead, is the state at time $\tau, \rho_{\tau}=U(\tau, 0) \rho_{i} U^{\dagger}(\tau, 0)$.

By using the definition of free energy, $F=\mathcal{U}-T S$, where $\mathcal{U}=\operatorname{tr}\{\rho H\}$ is the internal energy, while $S$ denotes the thermodynamic entropy (here evaluated as $S=$ $-\operatorname{tr}\{\rho \ln \rho\}$ for equilibrium states, at points $i, A$, and $B$ ), one can show that $\left\langle w_{\text {irr }}\right\rangle$ is related to the (average) heat required to let the system thermalize, starting from the state $\rho_{\tau}$, by keeping it in contact with a heat bath at temperature $T_{B}=\beta_{B}^{-1}$ (see also [17]):

$$
\left\langle w_{\mathrm{irr}}\right\rangle=T_{B}\left(S_{B}-S_{i}\right)-\left\langle Q_{\tau \rightarrow B}^{\mathrm{th}}\right\rangle,
$$

where $\left\langle Q_{\tau \rightarrow B}^{\text {th }}\right\rangle=\operatorname{tr}\left\{\left(\rho_{B}-\rho_{\tau}\right) H_{f}\right\}$ is the energy the system takes in the thermalization process leading it from the state $\rho_{\tau}$ to $\rho_{B}$ [19]. Thus, the irreversible work is related to both (i) the heat and the entropy change for the thermalization process $\tau \rightarrow B$, and (ii) the distance between the equilibrium state $\rho_{B}$ and the actual one, $\rho_{\tau}$.

Inner friction.-In the context of finite time thermodynamics, one is often led to compare the $i \rightarrow \tau$ process with the reversible adiabatic transformation $i \rightarrow A$ (rather than with the isothermal $i \rightarrow B$ ). Indeed, adiabatic transformations enter the Carnot and the Otto cycles and have been, therefore, largely studied so far [20]. In particular, when analyzing finite time adiabatic transformations performed on thermally isolated quantum systems, it is quite natural to introduce the concept of inner friction. This is defined as the nonadiabatic work; i.e., as the difference between the actual work performed on the system and the ideal one, done along an ideal reversible adiabatic transformation [21-24]. This difference comes in when the system is unable to adiabatically follow the control protocol, typically because some (inner or intrinsic) degrees of freedom do not commute with the control Hamiltonian.

As is the case for the irreversible work, we will show that inner friction too is related to a distance between the states attained through the hypothetical reversible and the actual unitary transformations, respectively. Furthermore, we will show that inner friction (and, in particular, the entropy production associated to it) can be described through a stochastic variable fulfilling a thermodynamic fluctuation relation.

Explicitly, in a reversible and quasistatic adiabatic transformation, the energy levels of the system experience a change as $H\left[\lambda_{i}\right]$ is slowly modified into $H\left[\lambda_{f}\right]$, but their occupation probabilities stay the same [25], so that, for every eigenstate $\left|\varepsilon_{m}^{(f)}\right\rangle$ of $H_{f}$, the population is still given by the initial value $P_{m}^{(i)}=\exp \left\{-\beta_{i} \varepsilon_{m}^{(i)}\right\} / Z\left[\lambda_{i}, \beta_{i}\right]$. If the energy eigenvalue is changed from $\varepsilon_{m}^{(i)}$ to $\varepsilon_{m}^{(f)}$ as $\lambda(t)$ goes from $\lambda_{i}$ to $\lambda_{f}$, this implies that also the temperature has changed. Its final value is such that $P_{m}^{(i)}=P_{m}^{(A)}$; that is $\exp \left\{-\beta_{i} \varepsilon_{m}^{(i)}\right\} / Z\left[\lambda_{i}, \beta_{i}\right]=\exp \left\{-\beta_{A} \varepsilon_{m}^{(f)}\right\} / Z\left[\lambda_{f}, \beta_{A}\right]$. The requirements for a reversible adiabatic process are indeed very tight as this relation has to hold for any adiabatically evolved eigenstate; that is, for every $m$. This, in turn, implies that all energy gaps of the system have to change by the ratio $\beta_{i} / \beta_{A}[28,29]$.

Under such conditions, the work performed on the $i \rightarrow A$ transformation is given by

$$
\left\langle w_{i \rightarrow A}\right\rangle=\mathcal{U}_{A}-\mathcal{U}_{i} \equiv \sum_{m} P_{m}^{(i)}\left(\varepsilon_{m}^{(f)}-\varepsilon_{m}^{(i)}\right) .
$$

This is, once again, different from the average work performed during the actual (unitary and finite-time) process $i \rightarrow \tau$. The difference between the two,

$$
\left\langle w_{\text {fric }}\right\rangle=\langle w\rangle-\left\langle w_{i \rightarrow A}\right\rangle,
$$

has been called inner friction as it is due to unwanted transitions that one would typically associate with heat. Indeed, by its definition and as shown by Eq. (10) below, the inner friction is precisely the "excess heat" the system has taken and that it would release to a thermal bath at inverse temperature $\beta_{A}$.

We now show that, similarly to Eq. (2) for the irreversible work, $\left\langle w_{\text {fric }}\right\rangle$ is given by the distance between the actual final state $\rho_{\tau}$ and the (hypothetical) equilibrium state $\rho_{A}=e^{-\beta_{A} H_{f}} / Z(A)$, evaluated through the quantum relative entropy

$$
\left\langle w_{\text {fric }}\right\rangle=\frac{1}{\beta_{A}} D\left(\rho_{\tau} \| \rho_{A}\right) .
$$

Indeed, by Eq. (4), one gets

$$
\begin{aligned}
\left\langle w_{\text {fric }}\right\rangle & =\langle w\rangle-\left\langle w_{i \rightarrow A}\right\rangle=\operatorname{tr}\left\{\rho_{\tau} H_{f}\right\}-\mathcal{U}_{A} \\
& =\sum_{m} \varepsilon_{m}^{(f)}\left[\left\langle\varepsilon_{m}^{(f)}\left|\rho_{\tau}\right| \varepsilon_{m}^{(f)}\right\rangle-P_{m}^{(A)}\right],
\end{aligned}
$$


while

$$
\begin{aligned}
D\left(\rho_{\tau} \| \rho_{A}\right) & =\operatorname{tr}\left\{\rho_{\tau} \ln \rho_{\tau}\right\}-\operatorname{tr}\left\{\rho_{\tau} \ln \rho_{A}\right\} \\
& =\sum_{m} P_{m}^{(i)} \ln P_{m}^{(i)}-\left\langle\varepsilon_{m}^{(f)}\left|\rho_{\tau}\right| \varepsilon_{m}^{(f)}\right\rangle \ln P_{m}^{(A)} \\
& =\sum_{m} \ln P_{m}^{(A)}\left[P_{m}^{(i)}-\left\langle\varepsilon_{m}^{(f)}\left|\rho_{\tau}\right| \varepsilon_{m}^{(f)}\right\rangle\right] \\
& =\sum_{m} \beta_{A} \varepsilon_{m}^{(f)}\left[\left\langle\varepsilon_{m}^{(f)}\left|\rho_{\tau}\right| \varepsilon_{m}^{(f)}\right\rangle-P_{m}^{(A)}\right],
\end{aligned}
$$

where we used $P_{m}^{(i)}=P_{m}^{(A)}$. These two relations, taken together, demonstrate Eq. (6).

Being given by a relative entropy, $\left\langle w_{\text {fric }}\right\rangle$ is thus always greater than zero by the Klein's inequality [30].

Furthermore, similarly to what has been done in Ref. [14], one can find a better (geometric) lower bound expressed in terms of the finite Bures length:

$$
\beta_{A}\left\langle w_{\text {fric }}\right\rangle \geq \frac{8}{\pi^{2}} \mathcal{L}^{2}\left(\rho_{\tau}, \rho_{A}\right),
$$

where, for any two density operators, $\mathcal{L}$ is given in terms of the fidelity $\mathcal{F}$ between those states, $\mathcal{L}\left(\rho_{1}, \rho_{2}\right)=$ $\arccos \left\{\sqrt{F\left(\rho_{1}, \rho_{2}\right)}\right\}$, with

$$
\mathcal{F}\left(\rho_{1}, \rho_{2}\right)=\left[\operatorname{tr}\left\{\sqrt{\sqrt{\rho_{1}} \rho_{2} \sqrt{\rho_{1}}}\right\}\right]^{2} .
$$

The inner friction is hence bounded from below by the geometric distance between the actual density operator $\rho_{\tau}$ at the end of the process and the corresponding equilibrium operator $\rho_{A}$, as measured by the Bures angle $\mathcal{L}$.

This gives a precise meaning to the idea that, when performing an adiabatic transformation in a finite time, the amount of work that "gets lost" is larger when the system is brought far and far away from equilibrium.

Going back to the thermalization process $\tau \rightarrow A$, we have that the average heat taken by the system to thermalize at $T_{A}=\beta_{A}^{-1}$ is given by

$$
\beta_{A}\left\langle Q_{\tau \rightarrow A}^{\text {th }}\right\rangle=-\beta_{A}\left\langle w_{\text {fric }}\right\rangle=-D\left(\rho_{\tau} \| \rho_{A}\right),
$$

which easily compares with the analogous expression for $\left\langle Q_{\tau \rightarrow A}^{\text {th }}\right\rangle$ reported in Eq. (3), as $S_{i}=S_{A}$ for an adiabatic process [31].

The heat exchange in a thermalization process is a quantity of fundamental interest as, through the Landauer principle, it is linked to information processing, storing, and erasing protocols, as well as the information-to-energy conversion [32]. Indeed, since any attempt at exploring the fundamental energetic limits of information processing would need to measure such a heat, this subject has been studied in various contexts [33].

Comparing the definitions of the two average heat exchanges, one obtains $\left\langle Q_{\tau \rightarrow A}^{\text {th }}\right\rangle-\left\langle Q_{\tau \rightarrow B}^{\text {th }}\right\rangle=\mathcal{U}_{A}-\mathcal{U}_{B}$, which gives an explicit relation between irreversible work and inner friction:

$$
\left\langle w_{\text {irr }}\right\rangle-\left\langle w_{\text {fric }}\right\rangle=\left(\mathcal{U}_{A}-\mathcal{U}_{B}\right)-T_{i}\left(S_{A}-S_{B}\right),
$$

or, stated differently, $\left\langle w_{\text {irr }}\right\rangle+F_{B}+T_{B} S_{i}=\left\langle w_{\text {fric }}\right\rangle+F_{A}+$ $T_{A} S_{i}$ [34].

Fluctuation relation.-For a reversible and infinitely slow $i \rightarrow \tau$ process, the actual final state $\rho_{\tau}$ would coincide with the equilibrium state $\rho_{A}$, with no net entropy change, as $S_{i}=S_{A}$. This latter equality implies that $\beta_{A} \mathcal{U}_{A}-\beta_{i} \mathcal{U}_{i}=\beta_{A} F_{A}-\beta_{i} F_{i}$.

In the actual finite time process, instead, the entropy production is nonzero, as unwanted transitions between adiabatic energy eigenstates may occur, as signaled by $\left\langle w_{\text {fric }}\right\rangle$. We can fully characterize the entropy production due to these diabatic transitions by defining an auxiliary entropic variable $s$, obtained (as by now usual) by a twomeasurement protocol in which energy is measured at the beginning and at the end of the process. Given the two outcomes (say $\varepsilon_{n}^{(i)}$ and $\varepsilon_{m}^{(f)}$, respectively), we can build the stochastic variable

$$
s=\beta_{A} \varepsilon_{m}^{(f)}-\beta_{i} \varepsilon_{n}^{(i)},
$$

which is distributed according to the probability density

$$
p(s)=\sum_{n, m} P_{n}^{(i)} P_{n \rightarrow m}^{(\tau)} \delta\left(s-\beta_{A} \varepsilon_{m}^{(f)}+\beta_{i} \varepsilon_{n}^{(i)}\right),
$$

with $P_{n}^{(i)}=Z_{i}^{-1} e^{-\beta_{i} \varepsilon_{n}^{(i)}}$ and $P_{n \rightarrow m}^{(\tau)}=\left|\left\langle\varepsilon_{m}^{(f)}|U(\tau, 0)| \varepsilon_{n}^{(i)}\right\rangle\right|^{2}$.

The average value of $s$ gives $\langle s\rangle=\beta_{A} \operatorname{tr}\left\{\rho_{\tau} H_{f}\right\}-\beta_{i} \mathcal{U}_{i}$, which, for a reversible quasistatic transformation, with $\rho_{\tau} \equiv \rho_{A}$, would give the sought combination of internal energies: $\beta_{A} \mathcal{U}_{A}-\beta_{i} \mathcal{U}_{i}$. Furthermore, a fluctuation relation similar to Eq. (1), can be obtained:

$$
\begin{aligned}
\left\langle e^{-s}\right\rangle & =\sum_{n, m} P_{n}^{(i)} P_{n \rightarrow m}^{(\tau)} e^{-\left(\beta_{A} \varepsilon_{m}^{(f)}-\beta_{i} \varepsilon_{n}^{(i)}\right)} \\
& =\frac{Z_{A}}{Z_{i}} \equiv e^{-\left(\beta_{A} F_{A}-\beta_{i} F_{i}\right)}
\end{aligned}
$$

This is a special case of a more general relation derived by Tasaki [35], which is of particular relevance here due to its relation with $\left\langle w_{\text {fric }}\right\rangle$. Indeed, by use of Jensen's inequality, this implies that

$$
\langle s\rangle \geq \beta_{A} F_{A}-\beta_{i} F_{i},
$$

which shows that the average entropy production in the actual process is always larger than zero

$$
\langle\Sigma\rangle:=\langle s\rangle-\left(\beta_{A} F_{A}-\beta_{i} F_{i}\right) \geq 0 .
$$

This latter quantity is easily shown to be related to the inner friction and to the corresponding relative entropy 


$$
\langle\Sigma\rangle \equiv \beta_{A}\left\langle w_{\text {fric }}\right\rangle \equiv D\left(\rho_{\tau} \| \rho_{A}\right) .
$$

In analogy to what has been done by Jarzynski in Ref. [8], where the cumulants of the work distribution have been related to the free energy difference $F_{B}-F_{i}$, we can show that the cumulants $C_{n}$ of the distribution for the variable $s$ are related to the combination of free energies $\beta_{A} F_{A}-\beta_{i} F_{i}$ as [36]

$$
-\left(\beta_{A} F_{A}-\beta_{i} F_{i}\right)=\sum_{n=1} \frac{(-1)^{n}}{n !} C_{n} .
$$

Finally, this implies that the inner friction can be expressed as a combination of the cumulants of order larger than 2 :

$$
\langle\Sigma\rangle=\beta_{A}\left\langle w_{\text {fric }}\right\rangle=\frac{C_{2}}{2}-\frac{C_{3}}{6}+\ldots,
$$

where $C_{2}=\left\langle s^{2}\right\rangle-\langle s\rangle^{2}$ is the variance, $C_{3}=\left\langle s^{3}\right\rangle-$ $3\left\langle s^{2}\right\rangle\langle s\rangle+2\langle s\rangle^{3}$, and so on.

Discussion.-We have shown that it is meaningful to consider the closeness to an ideal adiabatic transformation of an actual unitary evolution of a generic quantum system, driven out of equilibrium by changing in time a work parameter $\lambda$ entering its Hamiltonian. The comparison of the work done on the system in the two cases naturally leads to the concept of inner friction, which is related to the heat the system would release to a thermal bath, if thermalizing at the final temperature $\beta_{A}^{-1}$. Inner friction also comes out naturally when considering the statistics of the entropy irreversibly produced during the actual process, which satisfies a fluctuation relation analogous to the Jarzynski equality. Indeed, the average excess entropy, produced due to the irreversible nature of the actual process, precisely coincides with the inner friction (divided by the final temperature), which, therefore, can be expressed as a cumulant series.

Inner friction has been previously considered in the literature through simple microscopic models of thermal engines, where the working substance is composed of interacting spin dimers [22] or of a harmonic oscillator [37]. In the former, the friction comes from interaction; in the latter it comes from the intrinsic noncommutativity at different times of the oscillator Hamiltonian whose frequency is being varied. Further irreversible sources of noise and friction can be added artificially to the oscillator, such as frequency noise or phase noise [38].

Strategies against inner friction have been considered mainly under the generic names "shortcuts to adiabaticity" [39] [where control sequences $\lambda(t)$ are designed such that the irreversibility at the end of the adiabatic branch is minimized] and "quantum lubrication" [40] [where the coherences of $\rho_{\tau}$ in the $\left|\epsilon^{(f)}\right\rangle$ basis are minimized through an additional dephasing noise, thus minimizing $D\left(\rho_{\tau} \| \rho_{A}\right)$; see Eq. (8)].

It must be stressed though, that to the best of our knowledge, the irreversibility caused by inner friction had never been associated to a distance from an equilibrium state, nor to any fluctuation theorem.

As a final remark, we would like to emphasize once again the assumption on which our treatment relies, namely, the definition of $\beta_{A}$. The quantum adiabatic theorem $[25,27]$ guarantees that a very slow transformation would lead from the initial state $\rho_{i}=Z_{i}^{-1} \sum_{n} e^{-\beta_{i} \varepsilon_{n}^{(i)}}\left|\varepsilon_{n}^{(i)}\right\rangle\left\langle\varepsilon_{n}^{(i)}\right|$ to a final state with the same population and new eigenstates, $\rho_{A}=Z_{i}^{-1} \sum_{n} e^{-\beta_{i} \varepsilon_{n}^{(i)}}\left|\varepsilon_{n}^{(f)}\right\rangle\left\langle\varepsilon_{n}^{(f)}\right|$. We assumed this state to be a thermal equilibrium one at inverse temperature $\beta_{A}$. As mentioned above, this is a tight requirement that cannot always be fulfilled. There are, however, relevant cases in which there is no such problem: that of a harmonic oscillator (or of a harmonically trapped gas) and that of a two level system (or, more generally, a collection of noninteracting spins) whose frequency is parametrically changed during the process. For these systems, $\beta_{A}$ can always be defined, as well as for any other quantum system undergoing a transformation for which all of the initial energy gaps scale by the same factor. In all of these cases, our analysis is meaningful and the comparison of an actual unitary evolution with a reversible and quasistatic adiabatic transformation is well defined.

All the authors acknowledge support from COST MP1209 Action. F. P., G. F., and N. L. G. acknowledge insightful discussions with Michele Campisi, John Goold, and Mauro Paternostro. T. J. G. A. is supported by the European Commission, the European Social Fund, and the Region Calabria through the program POR Calabria FSE 2007-2013-Asse IV Capitale Umano-Obiettivo Operativo M2. F. G. and R. Z. acknowledge MINECO, CSIC, the EU commission, UIB and FEDER funding under Grants FIS2011-23526 (TIQS), postdoctoral JAE program (ESF) and Invited professors program.

[1] U. Seifert, Rep. Prog. Phys. 75, 126001 (2012).

[2] See, e.g., the experimental proposal in C. Bergenfeldt, P. Samuelsson, B. Sothmann, C. Flindt, and M. Büttiker, Phys. Rev. Lett. 112, 076803 (2014).

[3] M. Esposito, U. Harbola, and S. Mukamel, Rev. Mod. Phys. 81, 1665 (2009).

[4] C. Jarzynski, Annu. Rev. Condens. Matter Phys. 2, 329 (2011); M. Campisi, P. Hänggi, and P. Talkner, Rev. Mod. Phys. 83, 771 (2011).

[5] A. Polkovnikov, K. Sengupta, A. Silva, and M. Vengalattore, Rev. Mod. Phys. 83, 863 (2011).

[6] A. E. Allahverdyan, R. S. Johal, and G. Mahler, Phys. Rev. E 77, 041118 (2008); M. Esposito, R. Kawai, K. Lindenberg, and C. Van den Broeck, Phys. Rev. Lett. 105, 150603 (2010); G. Benenti, K. Saito, and G. Casati, Phys. Rev. Lett. 106, 230602 (2011); U. Seifert, Phys. Rev. Lett. 106, 020601 (2011); K. Brandner, K. Saito, and U. Seifert, Phys. Rev. Lett. 110, 070603 (2013); A. E. Allahverdyan, K. V. Hovhannisyan, A. V. Melkikh, and S. G. Gevorkian, Phys. Rev. Lett. 111, 050601 (2013). 
[7] M. Esposito and C. Van den Broeck, Phys. Rev. Lett. 104, 090601 (2010); S. Ciliberto, A. Imparato, A. Naert, and M. Tanase, Phys. Rev. Lett. 110, 180601 (2013); J. M. Horowitz and J. M. R. Parrondo, New J. Phys. 15, 085028 (2013).

[8] C. Jarzynski, Phys. Rev. Lett. 78, 2690 (1997).

[9] J. Kurchan, arXiv:cond-mat/0007360v2; S. Mukamel, Phys. Rev. Lett. 90, 170604 (2003).

[10] J. P. Pekola, P. Solinas, A. Shnirman, and D. V. Averin, arXiv: 1212.5808

[11] R. Dorner, S. R. Clark, L. Heaney, R. Fazio, J. Goold, and V. Vedral, Phys. Rev. Lett. 110, 230601 (2013); L. Mazzola, G. De Chiara, and M. Paternostro, Phys. Rev. Lett. 110, 230602 (2013); T. B. Batalhão, A. M. Souza, L. Mazzola, R. Auccaise, R. S. Sarthour, I. S. Oliveira, J. Goold, G. De Chiara, M. Paternostro, and R. M. Serra, Phys. Rev. Lett. 113, 140601 (2014); M. Campisi, R. Blattmann, S. Kohler, D. Zueco, and P. Hänggi, New J. Phys. 15, 105028 (2013).

[12] P. Talkner, E. Lutz, and P. Hänggi, Phys. Rev. E 75, 050102(R) (2007); P. Talkner and P. Hänggi, J. Phys. A 40, F569 (2007).

[13] G. E. Crooks, Phys. Rev. E 60, 2721 (1999).

[14] S. Deffner and E. Lutz, Phys. Rev. Lett. 105, 170402 (2010).

[15] F. Galve and E. Lutz, Phys. Rev. A 79, 032327 (2009); 79, 055804 (2009); A. Carlisle et al., arXiv:1403.0629.

[16] A. Silva, Phys. Rev. Lett. 101, 120603 (2008); F. N. C. Paraan and A. Silva, Phys. Rev. E 80, 061130 (2009); R. Dorner, J. Goold, C. Cormick, M. Paternostro, and V. Vedral, Phys. Rev. Lett. 109, 160601 (2012).

[17] T. J. G. Apollaro, G. Francica, M. Paternostro, and M. Campisi, arXiv:1406.0648.

[18] J. Yi, Y. W. Kim, and P. Talkner, Phys. Rev. E 85, 051107 (2012); A. Sindona, J. Goold, N. Lo Gullo, and F. Plastina, New J. Phys. 16, 045013 (2014).

[19] This relation comes directly from the definition of work, $\langle w\rangle=\operatorname{tr}\left\{\rho_{\tau} H_{f}\right\}-\mathcal{U}_{i}$, which implies $\langle w\rangle+\left\langle Q_{\tau \rightarrow B}^{\text {th }}\right\rangle=$ $\mathcal{U}_{B}-\mathcal{U}_{i} \equiv F_{B}-F_{i}+T_{B}\left(S_{B}-S_{i}\right)$. This is equivalent to Eq. (3), which, together with Eq. (2) gives $\beta_{B}\left\langle Q_{\tau \rightarrow B}^{\text {th }}\right\rangle=S_{B}-S_{i}-D\left(\rho_{\tau} \| \rho_{B}\right)$.

[20] M. J. Henrich, G. Mahler, and M. Michel, Phys. Rev. E 75, 051118 (2007); J. Birjukov, T. Jahnke, and G. Mahler, Eur. Phys. J. B 64, 105 (2008); H. T. Quan, Phys. Rev. E 79, 041129 (2009); A. M. Zagoskin, S. Savelév, F. Nori, and F. V. Kusmartsev, Phys. Rev. B 86, 014501 (2012); O. Abah, J. Roßnagel, G. Jacob, S. Deffner, F. Schmidt-Kaler, K. Singer, and E. Lutz, Phys. Rev. Lett. 109, 203006 (2012); J. Roßnagel, O. Abah, F. Schmidt-Kaler, K. Singer, and E. Lutz, Phys. Rev. Lett. 112, 030602 (2014).

[21] T. Feldmann and R. Kosloff, Phys. Rev. E 61, 4774 (2000).

[22] R. Kosloff and T. Feldmann, Phys. Rev. E 65, 055102(R) (2002); T. Feldmann and R. Kosloff, Phys. Rev. E 68, 016101 (2003); 70, 046110 (2004); 85, 051114 (2012).

[23] J. Wang, Z. Wu, and J. He, Phys. Rev. E 85, 041148 (2012).

[24] R. Wang, J. Wang, J. He, and Y. Ma, Phys. Rev. E 87, 042119 (2013).

[25] This is a result of the quantum adiabatic theorem [26]. We just mention here that a sufficient condition for this theorem to hold is the absence of level crossings. However, this is by no means necessary, as discussed, e.g., in Ref. [27] and references therein.
[26] A. Messiah, Quantum Mechanics (North Holland, Amsterdam, 1970).

[27] A. E. Allahverdyan and Th. M. Nieuwenhuizen, Phys. Rev. E 71, 046107 (2005).

[28] The conditions for a thermodynamic transformation to be a reversible adiabatic one have been established long ago; see, e.g., L. J. Broer, Physica (Amsterdam) 17, 531 (1951); M. J. Klein, Phys. Rev. 86, 807 (1952); R. D. Mountain, Physica (Amsterdam) 30, 808 (1964).

[29] H. T. Quan, Y.-X. Liu, C. P. Sun, and F. Nori, Phys. Rev. E 76, 031105 (2007).

[30] The non-negativity of the nonadiabatic work is the content of the minimal work principle, which is valid well beyond the cases we address here, as discussed in Ref. [27].

[31] As mentioned after Eq. (5), Eq. (10) qualifies the nonadiabatic work as a friction; that is, as an unwanted excess energy needed to perform the process, and giving rise to an excess heat the system releases when thermalizing.

[32] S. Toyabe, T. Sagawa, M. Ueda, E. Muneyuki, and M. Sano, Nat. Phys. 6, 988 (2010).

[33] M. Esposito and C. Van den Broeck, Europhys. Lett. 95, 40004 (2011); S. Deffner and C. Jarzynski, Phys. Rev. X 3, 041003 (2013); J. Goold, U. Poschinger, and K. Modi, Phys. Rev. E 90, 020101 (2014); J. Goold, M. Paternostro, and K. Modi, arXiv:1402.4499.

[34] We notice that Eq. (11) is equivalent to two explicit relationships among relative entropies, which read

$$
\begin{aligned}
& T_{B} D\left(\rho_{\tau} \| \rho_{B}\right)-T_{A} D\left(\rho_{\tau} \| \rho_{A}\right)=T_{B} D\left(\rho_{A} \| \rho_{B}\right) \\
& =-T_{A} D\left(\rho_{B} \| \rho_{A}\right)+\left(S_{A}-S_{B}\right)\left(T_{A}-T_{B}\right) .
\end{aligned}
$$

These two equalities hold for every process $\lambda_{i} \rightarrow \lambda_{f}$ bringing $\rho_{i}$ into $\rho_{\tau}$; that is, they hold for every final state $\rho_{\tau}$. Proof: each of the three sides entering Eqs. (18) and (19) is equal to $\mathcal{U}_{A}-\mathcal{U}_{B}+T_{B}\left(S_{B}-S_{A}\right)$ [as one can show by using Eq. (11) for the first term and by direct evaluation for the others].

[35] H. Tasaki, arXiv:cond-mat/0009244v2.

[36] A possible derivation of Eq. (16) goes as follows: Let $g(x)=\ln \left(\left\langle e^{-x s}\right\rangle\right)$ the generating function for the cumulants, with $C_{n}=\left.(-1)^{n}\left(d^{n} g / d x^{n}\right)\right|_{x=0}$. Then, $g(x)=$ $\sum_{n=1}\left((-1)^{n} / n !\right) C_{n} x^{n}$. Taking $x=1$, one obtains $g(1)=$ $\ln \left(\left\langle e^{-s}\right\rangle\right)=\sum_{n=1}\left((-1)^{n} / n !\right) C_{n}$, which gives Eq. (16).

[37] Y. Rezek and R. Kosloff, New J. Phys. 8, 83 (2006).

[38] E. Torrontegui and R. Kosloff, Phys. Rev. E 88, 032103 (2013).

[39] M. V. Berry, J. Phys. A 42, 365303 (2009); X. Chen, A. Ruschhaupt, S. Schmidt, A. del Campo, D. Guéry-Odelin, and J. G. Muga, Phys. Rev. Lett. 104, 063002 (2010); J.-F. Schaff, X.-L. Song, P. Vignolo, and G. Labeyrie, Phys. Rev. A 82, 033430 (2010); J.-F. Schaff, X.-L. Song, P. Capuzzi, P. Vignolo, and G. Labeyrie, Europhys. Lett. 93, 23001 (2011); M. G. Bason, M. Viteau, N. Malossi, P. Huillery, E. Arimondo, D. Ciampini, R. Fazio, V. Giovannetti, R. Mannella, and O. Morsch, Nat. Phys. 8, 147 (2012); N. Malossi, M. G. Bason, M. Viteau, E. Arimondo, R. Mannella, O. Morsch, and D. Ciampini, Phys. Rev. A 87, 012116 (2013); A. Del Campo, J. Goold, and M. Paternostro, Sci. Rep. 4, 6208 (2014); J. Deng, Q.-h. Wang, Z. Liu, P. Hänggi, and J. Gong, Phys. Rev. E 88, 062122 (2013).

[40] T. Feldmann and R. Kosloff, Phys. Rev. E 73, 025107(R) (2006). 